et al. Results from the first year of the New Zealand cot death study. NZ Med f 1991;104:71-6.

4 Mitchell EA, Taylor BJ, Ford RPK, Stewart AW, Becroft DMO, Thompson JMD, et al. Four modifiable and other major risk factors for cot death: the New Zealand Study. I Paediatr Child Health 1992;28(suppl 1):S3-8.

5 Breslow NE, Day NE. Statistical methods in cancer research. Vol 1. The analysi of case-control studies. Lyon: IARC, 1980:74-8, 133-4 (IARC Scientific Publication No 32)

6 Lee NNY, Chan YF, Davies DP, Lau E, Yip DCP. Sudden infant death syndrome in Hong Kong: confirmation of low incidence. BMF 1989;298 721

7 Beal SM. Sudden infant death syndrome: epidemiological comparisons between South Australia and communities with a different incidence. Aust Paediatr f 1986;22(suppl):13-6.

8 Black L, David RJ, Brouilette RT, Hunt CE. Effects of birth weight and ethnicity on incidence of sudden infant death syndrome. 7 Pediat 1986;108:209-14
9 Kraus JF, Greenland S, Bulterys M. Risk factors for sudden infant death syndrome in the US collaborative perinatal project. Int $f$ Epidemiol 1989;18: $113-20$.

10 Balarajan R, Raleigh VS, Botting B. Sudden infant death syndrome and post neonatal mortality in immigrants in England and Wales. BMF 1989;298: 716-20

11 Kyle D, Sunderland R, Stonehouse M, Cummins C, Ross O. Ethnic differences in incidence of sudden infant death syndrome in Birmingham. Arch Dis Child 1990;65:830-

12 Borman B, Fraser J, de Boer G. A national study of sudden infant death syndrome in New Zealand. NZ Med f 1988;101:413-5.

13 Tangermann R, McCarthy B, Schmidt E, Limbacher M. Zur hohen Nachsterblichkeit der Suglinge in NRW. Monatsschr-Kinderheilkd 1987; 135:679-85.

(Accepted 14 October 1992)

\title{
Sudden infant death syndrome: links with infant care practices
}

\author{
M Gantley, D P Davies, A Murcott
}

\begin{abstract}
Objectives-To investigate infant care practices in a small ethnic minority population within Britain that might suggest possible factors contributing to the low incidence of the sudden infant death syndrome in Asian populations.
\end{abstract}

Design-Ethnographic interviewing, a qualitative comparative method drawn from social anthropology.

Setting-Central Cardiff.

Subjects-Non-random sample of 60 mothers of Bangladeshi or Welsh ethnic origin and working or middle class occupational status, who had infants under one year old. None of the families interviewed had experienced a sudden infant death.

Results-Broad cultural contrasts emerged as a series of themes from the interview data: living patterns, family networks, sleeping patterns, and concepts of time and dependence.

Conclusion-Bangladeshi infants were cared for in a consistently rich sensory environment; Welsh infants, in contrast, were more likely to experience alternating periods of high and low sensory input. Long periods of lone quiet sleep may be one factor that contributes to a higher rate of sudden deaths in white than in Asian infants.

\section{Introduction}

Sudden infant death syndrome-the sudden, unexpected, and unexplained death of an apparently healthy baby-remains the single most important cause of death in the United Kingdom of children aged between 1 and 12 months.' Research on its aetiology has focused, at one end of the spectrum, on causal mechanisms-for example, inherited metabolic diseases ${ }^{2}$-and, at the other end, on infant care practices that may be potentially protective or harmful, such as sleeping position. ${ }^{3}$

There is now increasing evidence of both national and regional variation in incidence. The syndrome is, for example, very rare in Hong Kong. ${ }^{45}$ Within Great Britain, data from the Office of Population Censuses and Surveys for the years 1982-5 (classified by mother's country of birth) indicate significantly lower rates among babies of mothers born in Africa or Asia (India, Pakistan, or Bangladesh) than in those of mothers born in the United Kingdom or the Republic of Ireland. ${ }^{6}$ In Birmingham, for 1981-3, with ethnicity classified by mother's own report, incidence of deaths from the syndrome was found to be lowest among Asian babies and highest among Afro-Caribbeans, with white infants falling in between. ${ }^{7}$ In east London between 1987 and 1990 the largest ethnic minority population, Bangla- deshis, had rates approximately half those of the white majority (L Hilder, unpublished data). Among Asian populations in the United States (Asian in this case referring to Chinese, Japanese, Vietnamese, or Philippino, defined by mother's own report), the incidence of the sudden infant death syndrome increased with period of residence. ${ }^{8}$

British research also provides information on neonatal deaths for Asian and white infants. OPCS data show higher neonatal deaths among Bangladeshi babies in 1981-3, but comparable rates in 1984-8. In Birmingham the rate of sudden infant deaths was low in Asian infants but there were high rates of congenital malformations resulting in perinatal mortality. In east London neonatal mortality among white and Asian groups was broadly similar. This evidence is not sufficient to suggest that low rates of sudden deaths in Bangladeshi infants are achieved at the expense of high neonatal mortality. It does reinforce the need for detailed local statistics, with ethnicity recorded by personal report rather than by country of birth

The relatively low incidence of the sudden infant death syndrome among Bangladeshi babies in Britain represents something of a paradox, since many of these babies grow up in conditions that would predict a relatively higher incidence of the syndrome. ${ }^{9} 10$ These include poorer socioeconomic conditions, apparently crowded housing, and young mothers often with many children. This paradox has prompted research on the potential contribution of varying infant care practices to the prevention of deaths from this syndrome. Rather than retrospective studies of infants who have died or epidemiological analyses of the sudden infant death syndrome in specific population groups, our research offers a complementary perspective. Recognising that the apparently low incidence of the sudden infant death syndrome was difficult to explain wholly in terms of currently recognised risk factors, we sought to identify other differences in infant care practices that may contribute to the lower incidence of such deaths in Bangladeshi babies. For this reason we did not work with families who had lost an infant: instead we investigated the ordinary patterns of beliefs about infants and their care in two different populations, mothers of Welsh and Bangladeshi infants in Cardiff.

THEORETICAL BACKGROUND: AN ANTHROPOLOGICAL PERSPECTIVE

In 1991 the Lancet carried a series of articles on medical anthropology arguing that "medical anthropology has now emerged as a potential focus for those interested in explaining disease in terms of the patient's cultural context." This paper is part of the emergent contribution of medical anthropology, and presents an
School of Hygiene and

Tropical Medicine, Keppe

BMF 1993;306:16-20 
account of the theoretical background (drawn from biological anthropology) and the method (drawn from social anthropology) of a study of culturally varying infant care practices.

Medical anthropology occupies a middle ground between biological and social anthropology, biological anthropology specialising in human adaptation to specific environments and social anthropology in the associated cultural systems of belief (ideologies). Anthropology adopts a broad comparative perspective, drawing on both historical and cross cultural evidence: parts of medical anthropology narrow the focus to beliefs about the nature, cause, and treatment of illness within specific cultures.

Biological anthropology in the present context considers infants and their care within an evolutionary perspective, arguing that over the millions of years it has taken for humans to evolve, infant-parent contact was likely to have been virtually constant for at least the first year of life. McKenna, recognising the species specific nature of the sudden infant death syndrome and the relatively narrow time range of the majority of deaths, drew attention to both the relatively long period of physical dependence of the human infant and the ways in which caring practices vary with historical and cultural contexts. ${ }^{12} \mathrm{He}$ described human infants as being likely to experience problems in the regulation of breathing, such as those associated with the sudden infant death syndrome, because their anatomical and neurological immaturity coincides with a period of change that is specific to humans, that of the acquisition and use of language. Thus development of intentional crying and later of speech requiring sophisticated, learned control of breathing - characterised as "speech breathing"-poses a challenge that is unique to human infants.

The second part of McKenna's thesis posits that during this period of respiratory instability the infant's "evolutionary past" should not be forgotten..$^{1314}$ In other words, the practice of placing infants to sleep on their own, for relatively long periods of time, which is widespread in Western industrialised societies, is not only historically and culturally unique but also evolutionarily recent. McKenna suggests that infants sleeping alone lose the external sensory stimulation that may stabilise breathing. The mechanisms through which this stimulation occurs include rocking, chest movement, touch, and noise, all of which are concomitants of close proximity to other people and affect levels of arousal; mothers sleeping close to infants also contribute to the monitoring and control of temperature and chemical microenvironment (particularly carbon dioxide levels). Current experimental work with mothers and infants sleeping together in sleep laboratories is tracing the interaction of their patterns of breathing, and has found that in such conditions infants spend less time in deep stages of sleep. ${ }^{14} 15$

We understand an important pathophysiological mechanism underlying the sudden infant death syndrome to be a failure of respiratory control at a vulnerable stage of development. Disturbance to this delicate equilibrium by a number of different risk factors coinciding in a particular vulnerable baby may upset the regulation of breathing, leading sometimes to death. Epidemiological risk factors are somehow linked with destabilising influences on breathing, and it is through their avoidance or modulation-for instance through a change in the sensory environment of sleep ${ }^{13}$ -that risk of death can be reduced.

\section{Method}

As Janes and Stall have explained: "qualitative and quantitative measurement may inform the other at different stages of the research process. ${ }^{16}$ We used a qualitative social anthropological approach to explore the quantitative, epidemiological evidence of international and intranational variations in the incidence of sudden infant deaths. Social anthropology addresses cultural ideologies by using a variety of methods, including community observation and ethnographic interviewing. Ethnography-literally, textual description of particular cultures-also describes the techniques used to achieve an understanding of the beliefs and practices of specific groups, be they medical students ${ }^{17}$ or ethnic groups within Britain. Thus ethnographic interviewing describes an open ended unstructured approach designed to encourage informants not only to describe individual infant care practices but also to locate them within a broader ideology. The interviewer sets the framework for the interview-in this case mothers were asked to describe "a day in the life" of their infant-but allows the informant to determine the pace and order of the conversation, to select topics considered important. The interviewer uses a checklist to ensure that specific areas are covered, requesting explanations or introducing new topics as necessary.

Sixty such interviews have been completed-with an interpreter where necessary-with mothers of babies under 1 year old and living in Cardiff, south Wales (population $279055^{19}$ ). Each interview lasted about an hour and was audiotaped and transcribed. The sample comprised 20 Bangladeshi and 20 Welsh working class mothers and 20 middle class mothers (although we recognise Alam's argument ${ }^{18}$ that urban versus rural origin is a more appropriate distinction within the Bangladeshi population in Britain). A middle class group of 13 Welsh and seven Bangladeshi mothers was included to reflect class based variations in infant care practices. Four of the Bangladeshi mothers had been educated in Britain; all continued to live in extended households. Bangladeshi identity remained strong, only one mother preferring to describe herself as "British Asian." In terms of both household size and number of children per family ethnicity seemed more important than class, with 3.6 and 3.7 people in Welsh working class and middle class households respectively, and 6.7 and $7 \cdot 1$ in Bangladeshi working class and middle class households respectively; Welsh middle and working class families had 1.5 children on average, while Bangladeshi working class averaged 3.4 and middle class $2 \cdot 1$. Such figures do not of course reflect variation in household constitution: Bangladeshi households were typically extended either longitudinally (over three generations) or laterally (two or more brothers living with their wives and children).

These groups should not be regarded as representative of the Cardiff population: the 1991 census data records a Bangladeshi population of 1663 , some $0 \cdot 6 \%$ of the total. ${ }^{19}$ Our sample was non-random, Bangladeshi mothers being recruited first in view of their relatively small numbers. Mothers of Welsh infants of similar age were then recruited from the same areas of the city; they were thus living in similar accommodation and served by the same health professionals.

Bangladeshi mothers were chosen as a relatively recently arrived group of suitable demographic structure who had had comparatively little exposure to the infant care practices of the city in which they now live. The definition "Bangladeshi" refers to infants whose mothers were born in Bangladesh, and the few mothers who were born or grew up in Britain. "Welsh" is used to describe the ethnic origin of babies of parents who described themselves as either Welsh or English, but their infants as Welsh.

\section{ETHNIC MONITORING}

It should be noted that data on the ethnic origin of babies who died of the sudden infant death syndrome 
Bangladeshi babies benefit from constant social stimulation

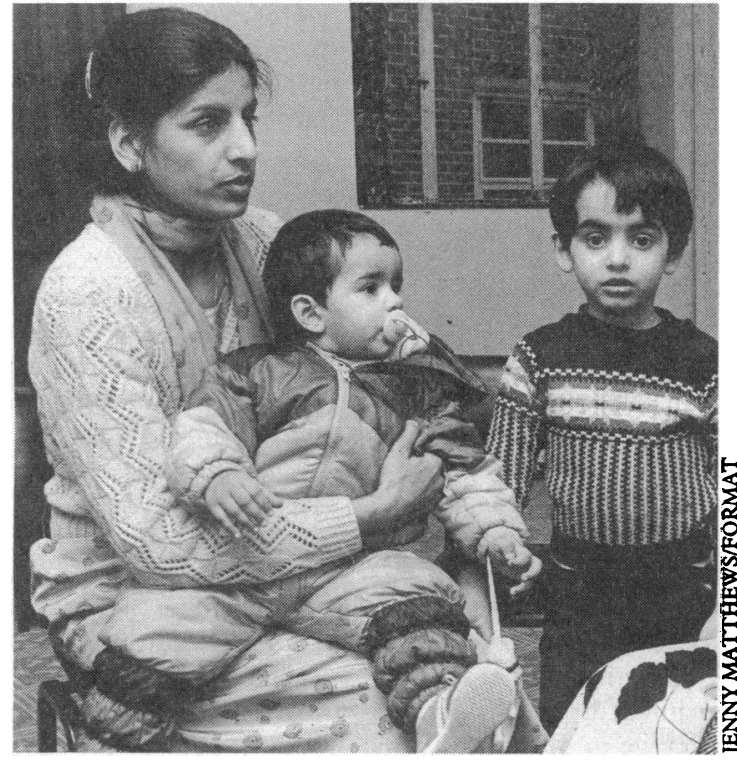

are collected only in certain areas, and the definitions used are inconsistent, being based either on mother's country of birth or her own report of her ethnicity. Classifying by mother's country of birth results in babies of, for instance, Pakistani mothers born in Britain appearing as British rather than Pakistani. Although such an attribution is important in terms of nationality, it loses information where health beliefs or possible variations in cultural practices are concerned. Consistent ethnic monitoring of both births and deaths is needed to provide accurate information on the local variations in the incidence of sudden infant deaths. This in turn will allow the recognition of high and low risk groups, and so the possibility of identifying high and low risk practices. If British born people of minority ethnic extraction are invisible in statistics on sudden infant deaths (or any other areas) we lose the chance to identify accurately both health trends and the health practices that may be associated with specific morbidity or mortality. The availability of the 1991 census data ${ }^{19}$ on local populations by ethnic origin offers a timely opportunity for ethnicity-like occupational class - to be systematically recorded.

\section{Results}

This paper reports broad cultural contrasts between Bangladeshi and Welsh mothers' beliefs and infant care practices. The evidence is presented as a series of themes which emerged from the interview data. The analytical procedure is to use each theme as an axis around which different elements of empirical data are grouped. ${ }^{20}$ They provide a context within which to understand the beliefs and practices of the Bangladeshi and Welsh mothers: this in turn allows practicespotential risk factors or protective factors-to be identified.

\section{LIVING PATTERNS}

Bangladeshis in Cardiff live in relatively large groups in small unheated Victorian terraced houses, adapting the pattern of extended family living to the accommodation available. A typical pattern is two or three brothers living together, each with a wife and several children. It presents a sharp contrast to similar houses occupied by their Welsh or English neighbours, divided into flats for nuclear units of mother-father-child or motherchild. The pattern of extended family living persists with the few Bangladeshis who have grown up in Cardiff or elsewhere in Britain. Bangladeshi infants are constantly in a busy social and tactile environment, whereas Welsh babies grow up in smaller households in which independence is encouraged. It is rare to leave a Bangladeshi baby to cry, or to play alone. Childcare is relatively public in Bangladeshi households, involving all the members of the extended family. In Welsh families, childcare is the prime responsibility of one or both parents, with occasional help from family, friends, and health professionals. Welsh parents attach considerable importance to privacy and to time away from the work of child care, for either work outside the home or leisure activities.

\section{FAMILY NETWORKS}

Adult Bangladeshis in Cardiff are children of large families and thus have extensive networks of cousins. Each of these is regarded as part of the family, as are people who originated in the same village. Family membership is highly valued, and new arrivals will be incorporated into groups resident in Britain, receiving practical and financial help if necessary. Bangladeshi grandparents in Cardiff live as part of the extended family, either sharing a house or living close by, and are closely involved in childrearing. The involvement of Welsh grandparents takes a different form: contact may be less frequent and based on invitations to visit or regular arrangements for contributing to childcare one day or one evening a week.

Bangladeshis in Cardiff continue to have large numbers of children, compared with Welsh and English families. The relatively large number of babies provides the opportunity for a more general familiarity with infants, and the arrival of a baby involves fairly small changes to family life. Thus very few preparations are made before birth. For the Welsh mothers, in contrast, the arrival of a baby often marks a series of dramatic changes associated with loss of income and employment, and the relatively low status, and often isolation, of motherhood. ${ }^{21}$

Most Bangladeshi mothers breastfed their babies for the first few weeks, switching to either combination or bottle feeding after this period. Bottle feeding was regarded as both more "Western" and "modern" and had the advantage that other family members could deal with feeding the infant. It is very rare for Bangladeshi women to smoke; some men do so. Smoking was more common among Welsh parents, accompanied by an attempt not to smoke while close to the baby. Oil is used to massage Bangladeshi babies after bathing, increasing their tactile stimulation; this task is often undertaken by grandmothers. Welsh mothers, in contrast, were more likely to use talcum powder.

\section{TIMES AND DATES}

Most Welsh households included husbands working outside the home during the day. Most of the Bangladeshi men worked in restaurants, so their working hours were more variable. They left the home between $4 \mathrm{pm}$ and $5 \mathrm{pm}$ and returned in the early hours of the morning, often coinciding with the feeding time for a young baby. The household timetable revolves around restaurant work, with children often being late for school, and visitors being least welcome (and this is a very hospitable culture) early in the morning. The notion of a routine, of a particular time for meals, baths, bed for young children-to fit in with the other interests and commitments of parents working outside the home-is not especially important. If Bangladeshi mothers' idea of time emphasises fluidity and flexibility, then Welsh mothers' notions emphasise regularity and routine.

\section{SLEEPING PATTERNS}

Bangladeshi babies are thought to be vulnerable, and they sleep close to other people both day and night; 
at night they are either in the mother's bed or in a cot next to it, a practice also reported by Farooqi et al..$^{22}$ They are put to sleep on their backs, being turned at times on to their sides in the belief that this will promote a culturally valued "rounded" head. Older children also sleep in their parents' room, a pattern which continued in the middle class Bangladeshi families in this sample and those in which the parents had grown up in Britain. It is not simply a question of availability of space, but of a belief that, as one mother put it, "I like to wake up in the night and see all my family around me."

The babies born to Welsh and English parents were, in contrast, sometimes placed in cots in their parents' rooms for a period of two to three months, and then encouraged (close to the peak age for the sudden infant death syndrome) to "get used" to sleeping alone, where possible in their own rooms. For daytime sleep Welsh babies were consistently said to need quiet, and to be placed in as peaceful a spot as possible, sometimes in another room, sometimes upstairs. In contrast, Bangladeshi babies sleeping during the day were always in the same room as other family members, in a relatively noisy, busy environment. There is less pressure in Bangladeshi families for babies to "sleep through the night." For Welsh parents, the demands of either or both working outside the home resulted in considerable emphasis on the infant's sleeping time.

Bangladeshi mothers expressed concern at the possibility of infant overheating, and this was one of the explanations given for the practice of shaving babies' heads. Welsh mothers were in general more concerned to keep an infant warm, although during the research period the publicity concerning the link between sudden infant death and temperature increased awareness of temperature, some mothers using room thermometers.

\section{INTERDEPENDENCE OR INDEPENDENCE}

All the mothers interviewed recognised that babies need looking after. In contrast to the notion of vulnerability in infants expressed by Bangladeshi families, there was among Welsh and English mothers a clearer push towards encouraging babies to be independent. This was motivated by the need for the mother to return to work or the wish for the parents to have time to themselves.

Among the Bangladeshis, the notion of interdependence of family members-both within and between generations-was clearer, underpinning the practices of parents and children sleeping in the same room and the extended family living together. Where Bangladeshi belief systems emphasise group membership and close informal proximity, Welsh belief systems emphasise individuality, independence, and self reliance.

\section{Discussion}

In the context of a varying incidence of the sudden infant death syndrome in different ethnic groups, and the possible contribution of infant care practices to this variation, this paper has described the beliefs and practices concerning infant care among two groups of carers in populations that have been shown to have significant differences in the likelihood of the syndrome. ${ }^{67}$

The themes presented describe the broad sociocultural context of the specific practices we identified. Some may protect against sudden infant deaths; others may add to risk. Some relate to factors that are already recognised (sleeping position, environmental temperature, smoking) and others offer new avenues for research.

During the fieldwork period, sudden infant deaths received enormous publicity in the national media. Both the Department of Health and the Foundation for the Study of Infant Deaths published leaflets describing the new advice that babies should be placed to sleep on their backs or sides rather than on their fronts. We considered the practical implications of our findings, and the potential contradictions faced by both parents and health professionals. The research process highlighted the difficulty of going from research to recommendations, and the effect of changing health advice on both health professionals providing advice and on mothers receiving it.

Inherent in this type of research is the potential to generate new categories or variables and to reconsider existing analytic categories in a fashion that also addresses their validity. An example arising from this study is the notion of infants being alone. Asking mothers to discuss whether or not their babies notice being on their own generated unfamiliar varieties of parental attitudes to children being left alone (for instance, to sleep in the day) or a report of the amount of time infants are likely to spend alone. One mother commented, while the baby was upstairs asleep, "she's never alone, someone's always here." Closer scrutiny of the precise meaning of "alone," as well as verification of the length of time infants would spend alone in a day, is needed if drawing conclusions that sudden infant deaths are not more common in babies whose periods of sleep are taken "on their own."

Reflecting on the rarity of the sudden infant death syndrome in crowded Hong Kong in 1985, Davies commented that "there might be some benefit to such high-density living. Babies are left alone much less. Sleep patterns might be different, effecting subtle modulations to physiological responses concerned with ventilatory control. The question 'When can I put baby into his own room?' is virtually never raised. Might closer overall contact with the sleeping baby somehow lessen the risks of sudden death?" 4 The environment in which Bangladeshi babies in Cardiff are brought up has similarities with that of Hong Kong, both being richer in sensory experiences ${ }^{1314}$ than that experienced by Welsh babies. We are not suggesting that to adopt all the practices evident among Bangladeshis in Cardiff, or Hong Kong residents, will prevent sudden infant deaths. In the words of Bergman, however, the sudden infant death syndrome "is like a nuclear explosion where a critical mass must be obtained before the event is to occur." ${ }^{23}$ This type of research represents a midpoint between the epidemiological linking of incidence in particular populations with particular infant care practices and the investigation of possible physiological mechanisms. It stands alone as a study of infant care practices, but we hope that it contributes to a discussion of those factors which form Bergman's "critical mass."

We acknowledge the generous financial support of the Foundation for the Study of Infant Deaths and the cooperation of the parents, health professionals, and interpreters who contributed towards this research.

1 Registrar General. Deaths by cause, sex and age in England and Wales 1990. London: HMSO, 1991. (OPCS series DH2 No 17.)

2 Holton JB, Allen JT, Green CA, Partington S, Gilbert RE, Berry JE. Inherited metabolic diseases in the sudden infant death syndrome. Arch Dis Child 1991;66:1315-7.

3 Wigfield RE, Fleming PJ, Berry PJ, Rudd PT, Golding J. Can the fall in Avon's sudden infant death rate be explained by changes in sleeping position? BMY 1992;304:282-3.

4 Davies DP. Cot death in Hong Kong: a rare problem? Lancet 1985;ii:1346-9.

5 Lee NNY, Chan YF, Davies DP, Lau E, Yip DCP. Sudden infant death in Hong Kong: confirmation of low incidence. $B M \mathcal{1}$ 1989;298:721.

6 Balarajan R, Raleigh VS, Botting B. Sudden infant death syndrome and palarajan R, Raleigh VS, Botting B. Sudden infant death syndrome and
postneonatal mortality in immigrants in England and Wales. BMJ 1989; postneonatal

7 Kyle D, Sunderland R, Stonehouse M, Cummins C, Ross O. Ethnic differences in incidence of sudden infant death syndrome in Birmingham differences in incidence of sudde.

8 Grether JK, Schulman J, Croen LA. Sudden infant death syndrome among Asians. F Ped 1990;116:525-8. 
Golding J, Limerick S, Macfarlane A. Sudden infant death: Patterns, pu 2 les and problems. Shepton Mallet: Open Books, 1985.

10 Southall DP, Samuels MP. Reducing risks in the sudden infant death syndrome. $B M F$ 1 1992;304:265.

1 Medicine and culture [editorial]. Lancet 1991;337:1012.

2 McKenna JJ. Evolution and sudden infant death syndrome (SIDS). Part I. Infant responsivity to parental contact. Human Nature 1990;1:145-77

13 McKenna JJ. Evolution and sudden infant death syndrome (SIDS). Part II. Why human infants? Human Nature 1990;1:179-206.

14 McKenna JJ, Mosko S. Evolution and Sudden Infant Death Syndrome (SIDS). Part III. Infant arousal and parent-infant co-sleeping. Human Nature 1990;1:291-330.

15 McKenna JJ, Mosko S, Dungy C, McAninch J. Sleep and arousal patterns of co-sleeping human mother/infant pairs: a preliminary physiological study co-sleeping human mother/infant pairs: a preliminary physiological study
with implications for the study of sudden infant death syndrome. Am f Phy's with implications for the stuc

16 Janes CR, Stall R, Gifford R. Anthropology and epidemiology: interdisciplinary approaches to the study of health and disease. Dordrecht: D Reidel, 1986.
17 Arkinson P. The clinical experience: an ethnography of medical education. Farnborough: Gower, 1981

18 Alam F. Salience of homeland: societal poliarization within the Bangladeshi population in Britain. Coventry: Centre for Research in Ethnic Relations, University of Warwick, 1988

9 Office of Population Censuses and Surveys. County Monitor 1991 Census, South Glamorgan. London: HMSO, 1991

20 Glaser BG and Strauss AL. The Discovery of grounded theory: strategies for qualitative research. Chicago: Aldine, 1967.

21 Oakley A. From here to maternity: becoming a mother. Harmondsworth: Penguin, 1981

22 Farooqi S, Perry IJ, Beevers DG. Ethnic differences in sleeping position and in risk of cot death. Lancet 1991;338:1455.

23 Bergman A. The discovery of sudden infant death syndrome: lessons in the practice of political medicine. New York: Praeger, 1986.

(Accepted 14 October 1992)

\title{
Investigation of inheritance of chronic inflammatory bowel diseases by complex segregation analysis
}

\author{
Marianne Orholm, Lennart Iselius, Thorkild I A Sørensen, Pia Munkholm, Ebbe Langholz, \\ Vibeke Binder
}

\section{Abstract}

Objective-To investigate the mode of inheritance of ulcerative colitis and Crohn's disease by complex segregation analysis.

Design-Cross sectional population based survey of familial occurrence of chronic inflammatory bowel disease.

Setting-Population of the Copenhagen county in 1987.

Subjects-662 patients in whom inflammatory bowel disease had been diagnosed before 1979, of whom $637(96 \%)$ provided adequate information. Of 504 patients with ulcerative colitis, 54 had 77 relatives with ulcerative colitis and of 133 patients with Crohn's disease, five had seven relatives with Crohn's disease.

Main outcome measures-Patterns of segregation of either disease as assessed by complex segregation analysis performed with the computer program POINTER.

Medical

Gastroenterological Department C, Herlev Hospital, University of Copenhagen, Copenhagen, Denmark

Marianne Orholm, senior registrar

Pia Munkholm, registrar Ebbe Langholz, research fellow

Vibeke Binder, chief physician

Department of Surgery, Karolinska Hospital, Stockholm, Sweden Lennart Iselius, registrar

Institute of Preventive Medicine, Copenhagen Health Services,

Copenhagen Municipal Hospital, Copenhagen Thorkild I A Sørensen, professor

Correspondence to: Dr M Orholm, Department of Infectious Diseases 7721,

Rigshospitalet,

Tagensvej 20, DK-2200

Copenhagen N, Denmark inheritance further we performed a complex segregation analysis. ${ }^{7}$ This allows testing of hypotheses of inheritance of a major dominant, additive, or recessive gene and multifactorial genetic or environmental inheritance.

\section{Patients and methods}

The county of Copenhagen has about 500000 inhabitants, about $10 \%$ of the total Danish population. The annual incidence of ulcerative colitis and Crohn's disease in this region was estimated for the years 1962 to 1978 and the prevalences on 31 December 1978 were calculated. ${ }^{8}$ Of the 694 patients with prevalent disease in 1978, 662 were alive on 1 January 1987. These patients served as probands in the present study.

The diagnostic criteria for ulcerative colitis and Crohn's disease in the probands have been described. ${ }^{68}$ Briefly, the diagnosis of ulcerative colitis was based on the presence of at least three of the following four criteria: a typical history of diarrhoea; stools containing blood and pus, or both, for more than one week or in repeated episodes; a typical sigmoidoscopic appearance, with granulated friable mucosa or ulcerations, or both; histological or cytological signs of inflammation; and radiological or colonoscopic signs of ulcerations with or without spiculation or granulation of the inner surface of the colon proximal to the rectum. The diagnosis of Crohn's disease was based on the presence of at least two of the following four criteria: a history of diarrhoea lasting more than three months; radiological findings of typical stenoses and prestenotic dilatation in the small bowel or segments with a cobblestone appearance in the large bowel; histological findings of transmural lymphocytic infiltration or occurrence of epithelial granulomas with giant cells of Langhans' type, or both; and the occurrence of fistulas or abscesses, or both, in a region of intestinal disease. Before either ulcerative colitis or Crohn's disease was diagnosed infectious and neoplastic diseases had to be ruled out.

In 10 patients who had met the criteria for ulcerative colitis in 1978 the diagnosis was later changed to Crohn's disease; the diagnosis was changed to ulcerative colitis in three patients who had originally had Crohn's disease diagnosed. The diagnoses presented in this study were those made in 1987-that is, the conclusion reached after a median $15(9-49)$ years of observation.

We sent a questionnaire requesting complete family history to each patient. Adequate information was 